\title{
Towards standards for light scattering studies of proteins stability and nanoparticle-protein interactions
}

\author{
Eleonora Frau and Silvia Schintke*, Member, IEEE \\ Laboratory of Applied NanoSciences (COMATEC-LANS), Department of Industrial Technologies, HEIG-VD \\ HES-SO // University of Applied Sciences Western Switzerland, CH-1401 Yverdon-les-Bains, Switzerland \\ e-mail: eleonora.frau@heig-vd.ch,silvia.schintke@heig-vd.ch
}

\begin{abstract}
Protein-nanoparticle suspensions are nowadays widely studied for the development of medical and environmental biosensors. The complexity of interactions between nanoparticles and biological fluids, together with the increasing use of dynamic light scattering (DLS) for their characterization, support the need to develop common standards for DLS measurements and analysis, in order to enable a reliable comparison of measurement results.

In this study, we use 3D cross-correlation Dynamic Light Scattering (3Dcc-DLS) for the characterization of gold nanoparticles (Au NP), with spherical and rod shape, stabilized with sodium citrate, and their interaction with bovine serum albumin (BSA). We show that for Au nanorods it is possible to distinguish the rotational and translational motion. Moreover, we monitor the interaction of Au NPs and BSA over time, in order to analyse the first step of protein corona formation. Our results show that Au nanorods interact more than Au nanospheres with BSA. From the evolution of the rotational and translational peaks we conclude that the proteins tend to bind on the long cylindrical side of the nanorods.
\end{abstract}

Keywords: dynamic light scattering, gold nanoparticles, bovine serum albumin, biomedical, protein corona.

\section{INTRODUCTION}

Gold nanoparticles (Au NPs) are widely used for biomedical applications, i.e. sensing, imaging and drug delivery, thanks to their unique photo-physical properties [1], [2]. In particular, anisotropic particle shapes like nanorods rise great interest due to the broad range for tuning their shape and size, impacting their optical and interaction properties [3] [4]. When Au NPs enter into contact with a biological fluid, a protein corona (PC) is formed around the surface, dominating the further interaction with other biological components [5]. In order to better understand how the PC formation takes place, our study is focused on the first stages of NPs-proteins interaction. We analyse four different $\mathrm{Au}$ NPs (nanospheres and nanorods with different sizes) and bovine serum albumin (BSA), a standard protein similar in composition and structure to human serum albumin (HSA). 3D-ccDLS is used to monitor the first minutes of the NP-BSA interaction. In view of the development of standards for DLS analysis, we propose a procedure to characterize NPs and proteins, and their interaction. This method allows to distinguish which NP the proteins have the tendency to bind with, and where on NPs' surface this binding is taking place.

\section{Experimental}

\subsection{Materials}

Four different aqueous suspensions of gold nanospheres (NSs) and nanorods (NRs), all stabilized by sodium citrate (NanoComposix), were used. Their main properties are presented in Table 1.

Table 1: Main properties of gold nanospheres and nanorods used in the present work; $D$ is the diameter, L the length, A.R. is the aspect ratio and $C$ is the initial concentration.

\begin{tabular}{|l|c|c|c|c|c|c|}
\hline Nanoparticles & Abbreviation & Shape & D $(\mathrm{nm})$ & L $(\mathrm{nm})$ & A. R. & C $(\mathrm{mg} / \mathrm{ml})$ \\
\hline Au NSs 40nm & NP1.0 & nanosphere & 40 & N.A. & 1.0 & 0.050 \\
\hline Au NRs abs 660 nm & NP2.6 & nanorod & 18 & 48 & 2.6 & 0.031 \\
\hline Au NRs abs 800 nm & NP3.6 & nanorod & 15 & 55 & 3.6 & 0.016 \\
\hline Au NRs abs 980 nm & NP5.6 & nanorod & 15 & 85 & 5.6 & 0.018 \\
\hline
\end{tabular}

Bovine serum albumin (BSA) in aqueous suspension, with a concentration of $2 \mathrm{mg} / \mathrm{ml}$, from Sigma Aldrich, was used as reference protein. To verify the stability, we have characterized it before and after filtration, with a $0.2 \mu \mathrm{m}$ pore size membrane.

Gold NPs and BSA suspensions were prepared at various concentrations $(\mathrm{C})$, in order to identify the suitable ranges for DLS measurements. For Au NPs we obtain stable DLS signals in a range from $0.016 \mathrm{mg} / \mathrm{ml}$ to 0.005 $\mathrm{mg} / \mathrm{ml}$, and for the filtered protein suspensions from $2 \mathrm{mg} / \mathrm{ml}$ to $0.76 \mathrm{mg} / \mathrm{ml}$.

\subsection{Optical characterization by dynamic light scattering and absorbance spectroscopy}

NPs and BSA were characterized by Modulated 3D cross-correlation Dynamic Light Scattering (3D-ccDLS) using a NanoLab 3D system (LS Instrument). The setup uses two laser beams (685 nm wavelength) and two detectors, 
temporally separated from each other, for the suppression of signal cross talk. High-speed intensity modulators, with a frequency of $5 \mathrm{MHz}$, and the scattering angle of $90^{\circ}$ are used. In this configuration multiple scattering events are significantly reduced [6], and even turbid samples can be measured [7]. The NP sizing is based on Brownian motion and Rayleigh scattering from particles which are significantly smaller than the laser wavelength. The scattered intensity is recorded as a function of time and the autocorrelation function is analysed. For spherical monodispersed particles the autocorrelation function decays exponentially, with a decay parameter proportional to the translational diffusion coefficient, $\mathrm{D}_{\mathrm{T}}$. According to the Stokes-Einstein equation (Eq. (1)), $\mathrm{D}_{\mathrm{T}}$ can be expressed as a function of the hydrodynamic radius, $\mathrm{R}_{\mathrm{H}, \mathrm{T}}$ :

$$
D_{T}=k T / 6 \pi \eta R_{H, T}
$$

where $\mathrm{k}$ is the Boltzmann constant, $\mathrm{T}$ the temperature, and $\eta$ the dynamic viscosity of the liquid. For non-spherical particles, like nanorods, the autocorrelation function is governed by two exponential decay parameters, one

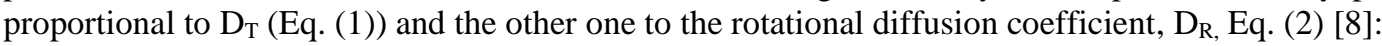

$$
D_{R}=k T / 8 \pi \eta R_{H, R}^{3}
$$

Our DLS data were acquired at a temperature of $20^{\circ} \mathrm{C}$. We compare results from analysis using Cumulants [9], and CORENN [10] algorithms. For both methods, we present the obtained $\mathrm{R}_{\mathrm{H}}$ values and the corresponding standard deviation of the particle size distribution. For statistics, we perform measurement series of 5 repetitions of $60 \mathrm{~s}$ for each concentration. For the study of Au-BSA interaction, we record time-dependent DLS series with 15 subsequent measurements of $60 \mathrm{~s}$ each. All experiments are performed at least twice for the confirmation of repeatability.

Optical absorbance spectra are recorded in the range from $200 \mathrm{~nm}$ and $1000 \mathrm{~nm}$ using an UV-vis-NIR spectrometer (OceanOptics, Flame, cuvette with $5 \mathrm{~mm}$ path). Both deuterium and halogen lamp were used.

\section{RESULTS AND DISCUSSION}

\subsection{Dynamic light scattering of gold nanoparticles and proteins}

We use 3D-ccDLS to characterize Au NPs, and Figure 1a shows the results from Cumulants algorithm, suitable for the analysis of spherical and monodispersed particles. The obtained radius $\mathrm{R}_{\mathrm{H}}=(28.5 \pm 0.17) \mathrm{nm}$ of the NP1.0 is in good agreement with the hydrodynamic diameter of $59.8 \mathrm{~nm}$ reported by the vendor. For the nanorods NP2.6, NP3.6 and NP5.6, Cumulants analysis lead to $R_{H}$ values of $(1.15 \pm 0.09) \mathrm{nm},(0.86 \pm 0.05) \mathrm{nm}$ and $(2.61 \pm 0.04) \mathrm{nm}$ respectively, which do not correspond to the physical nanorod dimensions. Thus, we apply also the CORENN algorithm, capable to analyse polydisperse samples; an example of size distributions at $0.005 \mathrm{mg} / \mathrm{ml}$ is shown Figure $1 \mathrm{~b}$. For the nanorods two main peaks are distinguished: the peak at large radius, $\mathrm{R}_{\mathrm{H}, \mathrm{T}}$, can be assigned to $D_{T}$, and the one at short radius, $R_{H, R}$, to $D_{R}[11]$. We note that for common non-spherical particles, rotational motion occurs in a very fast time scale, such that they are not detected by DLS. Au NRs, however, present birefringence [11], which renders their $D_{R}$ visible as a peak at small $R_{H}$.
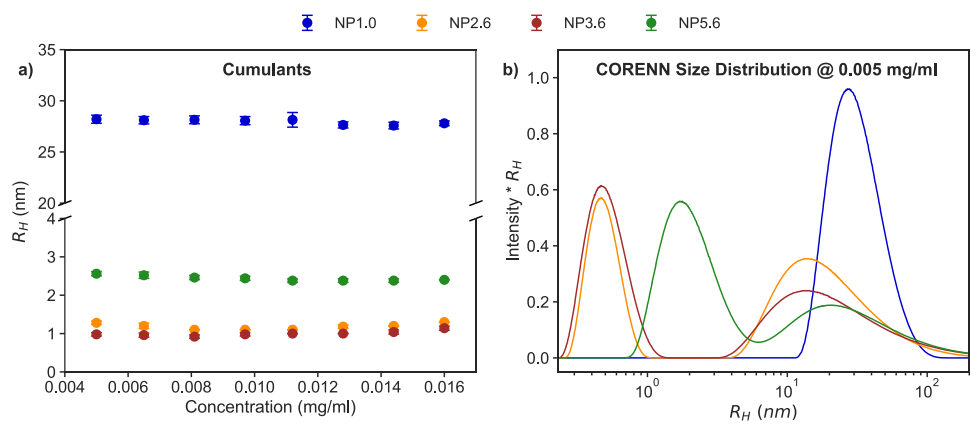

c)
\begin{tabular}{|l|r|r|r|}
\hline & $\begin{array}{c}\text { Cumulants } \\
\mathbf{R}_{\mathbf{0}}(\mathrm{nm})\end{array}$ & $\begin{array}{c}\text { CORENN } \\
\mathbf{R}_{\mathbf{0}-\mathrm{T}}(\mathrm{nm})\end{array}$ & $\begin{array}{r}\text { CORENN } \\
\mathbf{R}_{\mathbf{0}-\mathrm{R}}(\mathrm{nm})\end{array}$ \\
\hline $\mathbf{N P 1 . 0}$ & $28.50 \pm 0.17$ & $24.2 \pm 0.7$ & N.A. \\
\hline NP2.6 & $1.15 \pm 0.09$ & $8.7 \pm 0.3$ & $0.423 \pm 0.007$ \\
\hline NP3.6 & $0.86 \pm 0.05$ & $8.4 \pm 1.5$ & $0.401 \pm 0.010$ \\
\hline NP5.6 & $2.61 \pm 0.04$ & $13.6 \pm 1.4$ & $1.553 \pm 0.081$ \\
\hline
\end{tabular}

Figure 1: (a) Au NPs $R_{H}$ from Cumulants algorithm as a function of $C$; (b) size distributions from CORENN algorithm at $C=0.005 \mathrm{mg} / \mathrm{ml}$; (c) comparison of $R_{H}$ values at $C=0 \mathrm{mg} / \mathrm{ml}\left(R_{0}\right)$, obtained from analysis with both algorithms, extracted from linear regression.

The $y$-axis of Figure $1 \mathrm{~b}$ is chosen as the intensity (arbitrary units) of the distribution multiplied by the $\mathrm{R}_{\mathrm{H}}$, otherwise the intensity of the rotational peak significantly dominates over the translational one. In order to extract the peak positions, we analyse the mode of the original distributions as a function of concentration, using linear regression to evaluate the intercept $\left(\mathrm{R}_{\mathrm{H}}\right.$ at zero concentration, $\mathrm{R}_{0}$ ). The obtained results from Cumulants and CORENN analysis are summarized in Figure 1c. We note that the peak position of nanorods obtain from Cumulants and CORENN algorithm do not correspond to the physical dimensions (length and diameter) of NRs, but the observed $\mathrm{R}_{\mathrm{H}}$ values appear shorter. Since the rotational motion is faster than the translational one, it tends the distribution tends to shift towards smaller radii. It would be possible to use equations that relate the $\mathrm{R}_{\mathrm{H}}$ at zero 
concentration with the actual particle dimensions [8], [12], nevertheless these calculations are out of the main scope of this study.

We investigate also the BSA suspension at different concentrations, before and after filtration through a $0.2 \mu \mathrm{m}$ pore size membrane (Figure $2 \mathrm{a}-\mathrm{b})$.

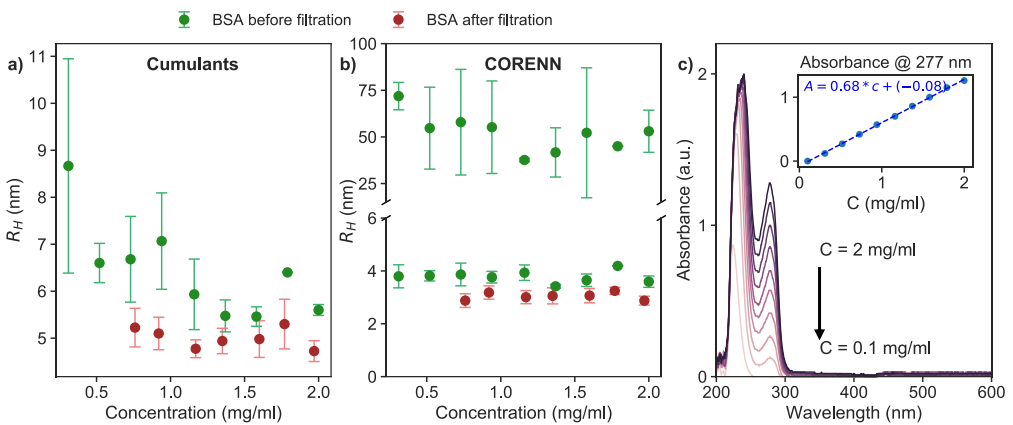

d)

\begin{tabular}{|l|c|c|}
\hline & $\begin{array}{c}\text { Cumulants } \\
\mathbf{R}_{\mathbf{0}}(\mathrm{nm})\end{array}$ & $\begin{array}{c}\text { CORENN } \\
\mathrm{R}_{\mathbf{0}}(\mathrm{nm})\end{array}$ \\
\hline $\begin{array}{l}\text { BSA before } \\
\text { filtration }\end{array}$ & $7.97 \pm 0.55$ & $3.82 \pm 0.18$ \\
\hline $\begin{array}{l}\text { BSA after } \\
\text { filtration }\end{array}$ & $5.22 \pm 0.29$ & $3.01 \pm 0.20$ \\
\hline
\end{tabular}

Figure 2: DLS results from Cumulants (a) and CORENN (b) and absorbance spectra (c) (calculated Beer-

Lambert low in the inset) of BSA suspensions as a function of $C ;(d)$ comparison of $R_{0}$ before and after filtration. We used $C(\mathrm{mg} / \mathrm{ml})=0.10 / 0.31 / 0.52 / 0.73 / 0.94 / 1.16 / 1.37 / 1.58 / 1.79 / 2.00$

Figure $2 b$ shows the presence of two peaks in the size distributions before filtration, while after filtration only one peak is detected, with a $\mathrm{R}_{\mathrm{H}}$ of BSA in good agreement with literature [13]. Thus, the peak at large $\mathrm{R}_{\mathrm{H}}$ before filtration is attributed to agglomerates. The $\mathrm{R}_{\mathrm{H}}$ values corresponding to the intercept at zero concentration are summarized in Figure 2d. For quantifying the concentration of filtered proteins, UV-vis-NIR absorbance spectra were recorded at various concentrations (Figure 2c). Two peaks are observed in the spectra, one in the far UV, at $210 \mathrm{~nm}$, and another in the near UV, at $280 \mathrm{~nm}$. The first one is associated to transitions in the polypeptide's backbone structure, and it is characteristic of the specific protein; the second one comes from the transition in the amino acids, and it is typically used to determine the protein concentration [14]. In the studied concentration range, the intensities of this second peak follow the Beer-Lambert law (Figure $2 \mathrm{c}$ inset), which we thus use to determine the concentration of the filtered protein suspensions.

\subsection{Gold nanoparticles and proteins interaction by dynamic light scattering}

Based on the above 3D-ccDLS studies, and on theoretical calculations of NP surface area available for the interaction with proteins, we have chosen to mix $0.8 \mathrm{ml}$ of $\mathrm{Au} \mathrm{NPs}$, at $\mathrm{C}=0.005 \mathrm{mg} / \mathrm{ml}$, with $16 \mu$ of filtered BSA, at $\mathrm{C}=1.97 \mathrm{mg} / \mathrm{ml}$, in order to study the PC formation. Figure 3 shows the results of time dependent 3D-ccDLS measurements for Cumulants and CORENN analysis. For the nanospheres NP1.0 the $\mathrm{R}_{\mathrm{H}}$ is not changing, thus they are not interacting with BSA under the studied conditions. To the contrary, for Au NRs we observe several features that indicate interaction with BSA. Firstly, $\mathrm{R}_{\mathrm{H}}$ from Cumulants increases with time, reaching a steady state value after about $5 \mathrm{~min}$ (Figure 3a), which is consistent with adsorption of BSA on NRs. Secondly, CORENN analysis shows an increase of $R_{H, R}$ over time (Figure $3 b$ ): the rotational movement is slowing down, in line with adsorption of BSA at the NRs surface. Also the $\mathrm{R}_{\mathrm{H}, \mathrm{T}}$ increases over time. Thus, to further elucidate the interaction, we determine the ratio of peak intensities (rotation/translation) from CORENN analysis of the BSA-NRs interaction over time (Figure 3c). This ratio is directly related to the aspect ratio of NRs [4]: the greater the aspect ratio, the greater the intensity ratio calculated from the size distribution. Figure $3 \mathrm{c}$ shows that intensity ratio decreases over time, thus the hydrodynamic diameter of NRs increases, leading to the conclusion that proteins adsorb at the circumference of the NRs.
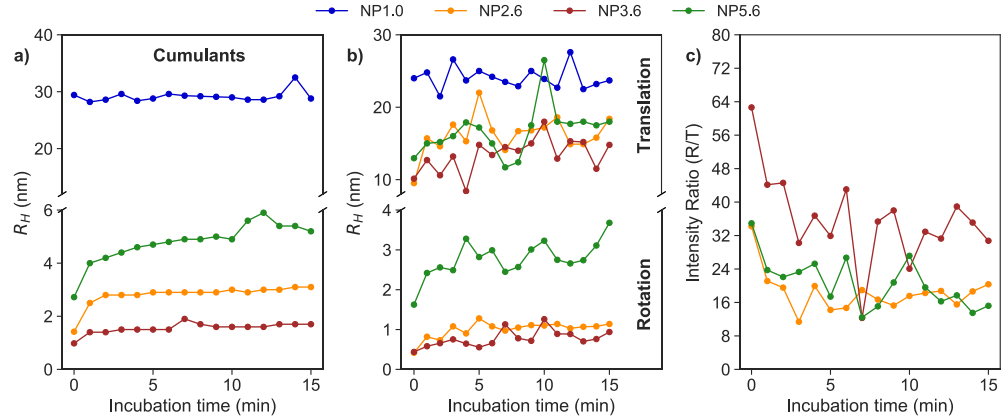

Figure 3: 3D-ccDLS results from Au NPs and BSA interaction. a) Cumulants results, b) CORENN results, were translational and rotational peak are distinguished, $c$ ) intensity ratio (rotation/translation) for the nanorods. 0.8 $\mathrm{ml}$ of Au NPs suspension, with $C=0.005 \mathrm{mg} / \mathrm{ml}$, are mixed with $16 \mu \mathrm{l}$ of BSA at $C=1.97 \mathrm{mg} / \mathrm{ml}$. 
Concerning the $\mathrm{R}_{\mathrm{H}, \mathrm{T}}$, the observed increase is less pronounce than the one of $\mathrm{R}_{\mathrm{H}, \mathrm{R}}$ (Figure $3 b$ ). Indeed, from Eq. (1) and Eq. (2) it follows that the $D_{R}$, and thus the corresponding radius, is more affected by shape changes, since it depends on $\mathrm{R}^{3} \mathrm{H}$.

In order to further understand the mechanism of PC formation, it would be interesting to monitor over time also chemical parameters, such as capping layer and zeta potential. Indeed, it has been reported [3], [4], [15], that these properties have also an influence in the aggregation of proteins around NPs.

\section{CONCLUSIONS}

We have used 3D cross-correlation dynamic light scattering to monitor the formation of protein corona, during the interaction between bovine serum albumin and gold nanoparticles of different shapes. For gold nanorods, we can distinguish the rotational and translational motion of the particles in the analysed size distributions. We have investigated the time evolution of the intensity ratio of rotational over translational peaks during protein corona formation. Since it decreases over time, we conclude that the protein corona formation is taking place on the cylindrical surface of gold nanorods. The applied analysis method can contribute to the establishment of standard analysis procedures of DLS data for time-dependent NPs interactions.

\section{ACKNOWLEDGEMENTS}

This work has been supported by the HES-SO programme P3, project HENAPAMAT IA-EXT18-22-90667.

\section{REFERENCES}

[1] E. C. Dreaden, A. M. Alkilany, X. Huang, C. J. Murphy and M. A. El-Sayed, "The golden age: gold nanoparticles for biomedicine," Chem. Soc. Rev., vol. 41, no. 7, pp. 2740-2779, 2012.

[2] E. Boisselier and D. Astruc, "Gold nanoparticles in nanomedicine: preparations, imaging, diagnostics, therapies and toxicity," Chem. Soc. Rev., vol. 38, pp. 1759-1782, 2009.

[3] S. Alam and A. Mukhopadhyay, "The conjugation of gold nanorods with bovine serum albumin protein," $J$. Phys. chem. C, vol. 118, no. 47, p. 27459-27464, 2014.

[4] H. Liu, N. Pierre-Pierre and Q. Huo, "Dynamic light scattering for gold nanorod size characterization and study of nanorod-protein interactions," Gold Bull, vol. 45, pp. 187-195, 2012.

[5] J. Liu and Q. Peng, "Protein-gold nanoparticle interactions and their possible impact on biomedical applications," Acta Biomaterialia, vol. 55, pp. 13-27, 2017.

[6] I. Block and F. Scheffold, "Modulated 3D cross-correlation light scattering: improving turbid sample characterization," Review of Scientific Intruments, Vols. 81, 123107, 2010.

[7] E. Frau and S. Schintke, "Modulated 3D cross-correlation dynamic light scattering of magnetic nanoparticle inks," Transparent Optical Networks (ICTON), 20th International Conference on IEEE, pp. 1-4, 2018.

[8] R. Pecora, "Dynamic light scattering measurement of nanometer particles in liquid," J. of Nanoparticle Research, vol. 2, pp. 123-131, 2000.

[9] B. Frisken, "Revisiting the method of cumulants for the analysis of dynamic light-scattering data," Appl. Opt., vol. 40, pp. 4087-4091, 2001.

[10] "DLS data analysis: the CORENN method," [Online]. Available: https://lsinstruments.ch/en/technology/dynamic-light-scattering-dls/corenn. [Accessed July 2020].

[11] P. Arenas-Guerrero, Á. V. Delgado, K. J. Donovan, K. Scott, T. Bellini, F. Mantegazza and M. L. \& Jiménez, "Determination of the size distribution of non-spherical nanoparticles by electric birefringence-based methods," Scientific Reports, vol. 8, no. 1, 2018.

[12] M. Tirado, "Comparison of theories for the translational and rotational diffusion coefficients of rod-like macromolecules. Application to short DNA fragments," J. Chem. Phys., vol. 81, p. 2047, 1984.

[13] S. Li, D. Xing and J. Li, "Dynamic light scattering application to study protein interactions in electrolyte solutions," Journal of Biological Physics, vol. 30, pp. 313-324, 2004.

[14] Sonu, H. Sayantan, K. Sunita, A. Rishika, V. K.A. and S. K.S., "Study on interactions of cationic gemini surfactants with folded and unfolded bovine serum albumin: Effect of spacer group of surfactants," Journal of Molecular Liquid, vol. 243, pp. 369-379, 2017.

[15] J. Piella, N. Bastús and V. Puntes, "Size-dependent protein-nanoparticle interaction in citrate-stabilized gold nanoparticles: the emergence of the protein corona," Bioconjugate Chem., vol. 28, no. 1, pp. 88-97, 2017. 